\title{
Масс-спектрометрия как современный метод анализа полимеров
}

\author{
(С)2021 Пыцкий И.С., Кузнецова Е.С., Буряк А.К. \\ Институт физической химии и электрохимии имени А.Н. Фрумкина РАН, Москва
}

Поступила в редакцию 9.10.2020 г.

DOI: $10.17308 /$ sorpchrom.2021.21/3221

Работа посвящена изучению места современных масс-спектрометрических методов в области исследования полимеров. Исследованы вопросы развития методов и показано, что практически до конца XX века масс-спектрометрия применялась для изучения полимеров крайне ограниченно из-за нелетучести полимеров. Рассмотрен метод пиролитической масс-спектрометрии как наиболее распространённый для определения молекулярно-массового распределения, определения концевых групп и других общих задач. Также описаны более современные методы “мягкой” ионизации, позволяющие ионизировать полимеры из конденсированного состояния с одновременной десорбцией в газовую фазу. Обзор литературы показал, что подобные методы практически полностью вытеснили или значительно дополнили инструментарий исследователя полимерных материалов. В работе со ссылками на литературу описаны матрично-активированная лазерная десорбция/ионизация (MALDI), полевая десорбция (FD), бомбардировка быстрыми атомами (SIMS), ионизация электораспылением (ESI). Также в качестве более классического метода описана пиролитическая масс-спектрометрия с прямым вводом и в сочетании с газохроматографическим разделением. Показано, что масс-спектрометрия наиболее универсальный и чувствительный метод при любых задачах в области анализа и исследования полимерных материалов.

Ключевые слова: масс-спектрометрия, полимеры, пиролиз, газовая хроматография, «мягкая» ионизация, полевая десорбция, масс-спектрометрия вторичных ионов, поверхность.

\section{Введение}

Масс-спектрометрия отличается от других методов исследования полимеров прежде всего высокой чувствительностью и быстротой анализа. Если на заре химии полимеров для их исследования применялись в основном классические спектральные методы (КР, УФ, ИК), то сейчас основной упор делается именно на методы масс-спектрометрии. В отличие от указанных выше методов, масс-спектрометрическая ионизация вызывает деструкцию исходной молекулы и молекулярный ион не всегда можно увидеть в масс-спектре. Однако именно это обстоятельство в сочетании с высокой чувстви- тельностью позволяет не только идентифицировать компоненты, но и охарактеризовать их и изучить природу их появления. Долгое время исследователи видели большие перспективы для использования масс-спектрометрии в области исследования полимеров, однако необходимость перевода образца в газовую фазу делала это невозможным. Революция в исследовании полимеров масс-спектрометрическими методами произошла после разработки методов ионизации в конденсированном состоянии с одновременной десорбцией образца в газовую фазу. Первыми из них были бомардировка быстрыми атомами (fast atom bombardment, FAB) и десорбция полем 
(field desorption, FD). Появление этих методов позволило исследовать олигомеры массами до нескольких тысяч Дальтон. Однако в настоящее время эти методы практически полностью заменены более современными: матрично-активированной лазерной десорбцией/ионизацией (matrix assisted laser desorption/ionization, MALDI), электрораспылительная ионизация (electrospray ionization, ESI) и вторично-ионная масс-спектрометрия (secondary ion mass spectrometry, SIMS). Эти методы оказались наиболее подходящими для исследования полимеров, так как позволяют ионизировать и десорбировать без значительного разрушения полимеры высоких масс, а благодаря комбинациям современных масс-анализаторов возможно проведение экспериментов по распаду и взаимодействию десорбированных молекул. На смену широко применявшимся в XX веке секторным и квадрупольным масс-анализаторам, которые имели верхний предел разделения по массам не более тысячи Дальтон, пришли времяпролётные анализаторы (time-offlight, TOF) с теоретически беспредельным диапазоном разделяемых масс. В последнее время они стали применятся в сочетании с масс-анализаторами сверхвысокого разрешения, которые позволяют определять точную массу и как следствие структуру полимера с массой более миллиона Дальтон. Это прежде всего орбитальная ловушка (orbit trap, OT) и анализатор ион-циклотронного резонанса с преобразованием Фурье (ion cyclotronic resonance - Fourier transform, ICR-FT). Комбинация этих анализаторов позволяет в полной мере использовать все преимущества масс-спектрометрии при анализе полимеров. Далее будут разобраны наиболее часто применяемые методы и показаны их преимущества и недостатки.

\section{Пиролитическая масс-спектрометрия}

Данный метод широко используется в различных областях науки и промышленности и представляет собой сочетание пиролитической ячейки с масс-спектрометром. Благодаря пиролизу, полимер распадается на мономерные звенья в результате программируемого нагрева. Этот метод широко используется для установления мономерного звена полимера. Особенно следует отметить его широкое распространение в комбинации с газовой хроматографией. Так в работе [1] метод применяется для исследования полимеров полиэтилена и полистирола. Целью работы было оптимизировать, а затем проверить метод Ру-GC/MS, определить предел обнаружения (LOD) для восьми распространенных полимеров. Оптимизация по нескольким параметрам ГХ была проведена с использованием микросфер из полиэтилена (ПЭ) и полистирола (ПС). Оптимизированный метод Py-GC/MS требует температуры пиролиза $700^{\circ} \mathrm{C}$, соотношения разделения 5 и 300 С в качестве температуры инжектора. Оценка производительности была проведена путем выполнения тестов на повторяемость и промежуточную точность, а также расчета предела обнаружения (LOD) для обычных полимеров. LOD все были ниже 1 мкг. Было проведено сравнение идентификаций, выполненных с помощью рамановской микроспектроскопии. Наконец, оптимизированный метод был применен к образцам окружающей среды, включая пластмассы, отобранные с поверхности морской воды и с пляжей. Показано, что метод является дополнением к $\mu$-рамановской спектроскопии. Большинство исследований полимеров связано с экологической тематикой и опять здесь становится незаменимой пиролитическая масс-спектрометрия. В работе [2] проводилось исследование пластиков извлечённых из желудка рыб. Поедание рыбами микропластика зарегистрировано у пресноводных и морских видов. В то время как многочисленные исследования позволили количественно определить и охарактеризовать микропластические частицы, аналитические методы идентификации полимеров ограничены. В этом исследовании изучалась 
применимость метода для идентификации микропластиков, извлеченных из содержимого желудков морской рыбы с побережья Техасского залива. Всего было проанализировано 43 микропластических частицы, включая 30 волокон, 3 фрагмента и 10 сфер. Поливинилхлорид (ПВХ) и полиэтилентерефталат (ПЭТ) были наиболее часто определяемыми полимерами (44.1\%), за ними следовали нейлон $(9.3 \%)$, силикон $(2.3 \%)$ и эпоксидная смола $(2.3 \%)$. Примерно $42 \%$ образцов не смогли отнести к определенному классу полимеров из-за ограниченного образования пиролитических продуктов, низкого содержания продукта или отсутствия сравнительных стандартов. Диэтилфталат, известный пластификатор, был обнаружен в $16.3 \%$ от общего количества образцов, включая ПВХ (14.3\%), силикон $(14.3 \%)$, нейлон $(14.3 \%)$ и неизвестные образцы (57.2\%). Однако определение молекулярно- массового распределения полимеров - не единственная возможность для пиролитической массспектрометрии. В работе [3] определяли концевые группы в серии образцов сополимера стирола - бутилакрилата в молярном соотношении сырья 80:20, полученных радикальной полимеризацией с различными количествами Пероксида бензоила в качестве инициатора. Два типа концевых групп, происходящих от инициатора, бензоилокси и концевые фенильные группы - были индивидуально определены с помощью ступенчатой газовой хроматографии с использованием печного пиролизера с двойной продувкой. На первой стадии (хемолиз) образец сополимера смешивали с гидроксидом тетраметиламмония и вводили в пиролизер при $250^{\circ} \mathrm{C}$ в течение 10 мин. Во время этой стадии бензоилоксиконцевые группы селективно и количественно разлагались на метилбензоат посредством термического гидролиза и метилирования сложноэфирных связей, что четко наблюдалось на пирограмме. На втором этапе образец остаточного полимера анализировали с помощью обычной Рy-GC при $600^{\circ} \mathrm{C}$. На наблюдаемой пирограмме бензол и $\mathrm{C}_{14} \mathrm{H}_{12}$ были отдельно обнаружены как ключевые продукты, отражающие концевые фенильные группы среди различных пиролизатов основной цепи полимера. На основании пиковой интенсивности этих характерных продуктов относительное содержание обоих типов концевых групп из инициатора было оценено как почти пропорциональное количеству в исходной смеси. Более того, было определено, что количество концевых бензоилокси-групп было примерно в два раза больше, чем количество концевых фенильных групп. Из этого небольшого обзора видно масштаб и применимость пиролитической масс-спектрометрии в современных исследованиях полимеров. Следует также отметить несколько современных обзоров [4-5] по данному вопросу охватавающих широкий круг проблем в области техники и синтеза полимерных материалов.

\section{Матрично-активированная лазерная десорбция/ионизация}

MALDI - один из наиболее популярных «мягких» методов ионизации применяемый для изучения высокомолекулярных соединений как природных, так и промышленных. В большинстве случаев применяется с времяпролётным массанализатором, обеспечивающим широкий диапазон пропускаемых масс. Возможны также комбинации с анализаторами сверхвысокого разрешения. Ионизация проводится импульсным лазером с поверхности, на которой закристаллизовано исследуемое соединение в смеси с соединением-матрицей, перераспределяющей излучение для мягкой ионизации (рис. 1)

Например, в работе [6] МАЛДИ используется в сочетании с новейшей техникой хроматографического разделения на двух последовательных колонках с различным механизмом разделения. Были исследованы структура и состав сополимера полидиметилсилоксана и 


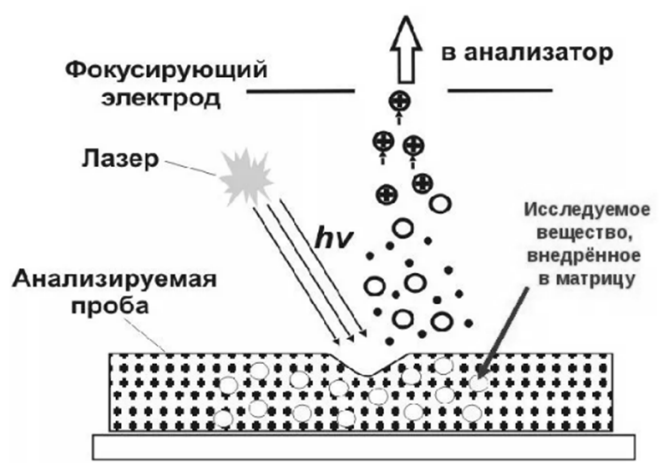

Рис.1. Схема ионизации методом матрично-активированной лазерной десорбции/ионизации

Fig. 1. The scheme of matrix-assisted laser desorption/ionization

поликарбоната с применением различных аналитических подходов, включая методы хроматографического разделения, спектрометрические и спектроскопические методы обнаружения. В частности, эксклюзионная хроматография и жидкостная адсорбционная хроматография, работающие в различных условиях, были использованы для достижения разделения в соответствии с молярной массой и распределением функциональности. Объединение обоих методов привело к созданию двумерных графиков, которые можно было использовать для простого сравнения различных партий сополимеров. Для структурных исследований применялась матричная лазерная десорбция/ионизация-времяпролетная массспектрометрия (MALDI-TOF). Полученная методология позволила отличить сополимер от гомополимерных цепей ПК, присутствующих в материале. В 2017 году была защищена диссертация, полностью посвящённая вопросам исследования поверхностных неоднородностей полимеров [7]. Состав смеси полимеров на поверхности обычно отличается от состава в объеме, и градиент состава с глубиной имеет важное значение для свойств поверхности. Определение состава поверхности представляет различные проблемы, которые побуждают к разработке новых методов количественного определения состава. В работе метод времяпролетной масс-спектрометрии с лазерной десорбцией и ионизацией с ис- пользованием матрицы получил дальнейшее развитие для решения конкретных вопросов поведения поверхности полимерной смеси при определении состава поверхности. .

Первый вопрос заключается в том, как несоответствие длины цепи влияет на поверхностную сегрегацию в случае, если несоответствие довольно мало. Для полистирола 6 кДа среднечисловая молекулярная масса (Mn) на поверхности составляет где-то на 300 Да ниже, чем в основной массе, а для полиметилметакрилата 7 кДа сдвиг составляет около 500 Да.

Второй вопрос заключается в том, как функционализация конца цепи влияет на преобладание на поверхности любой части цепи, имеющей функционализацию конца. Показано, что поверхность смеси полистирола 6 кДа и полистирола 6 кДа, функционализированного гидроксиметильными концами, не только обеднена концевыми группами с более высокой энергией, но и лишена любых сегментов, принадлежащих функционализированным цепям. Это демонстрируется с помощью MALDI-ToF-MS. Это исследование было продолжено на поверхности смесей полистирола 6 кДа и полистирола 6 кДа, функционализированных гидроксиэтильными концами.

Также проблема определения латеральных изменений в составе поверхности была решена благодаря использованию визуализации MALDI-ToF-MS.

Отдельно следует отметить работы, в 
которых MALDI используется для контроля процессов синтеза полимеров. Например, в работе [8] использовали группу методов масс-спектрометрии с высоким разрешением, диссоциацию столкновениями и спектроскопию ионной подвижности и реализовали структурную характеризацию побочных полимерных продуктов, обнаруженных вместо интактных частиц при выполнении матричной лазерной десорбции/ионизации (MALDI) полистиролов с малостабильными концевыми группами. Исследуемые образцы были получены методами радикальной полимеризации с переносом атома и обратимой полимеризацией с переносом фрагментации. Спектральное разрешение, обеспечиваемое ортогональной инжекцией ионов MALDI в рефлектронный времяпролетный массанализатор, позволило провести тщательную проверку типов концевых групп, в том числе некоторых с одинаковым номинальным значением $\mathrm{m} / \mathrm{z}$, но с различным элементным составом. Определение массы индивидуальных концевых групп было достигнуто в экспериментах MC/MC. Помимо подтверждения общеизвестных полистирольных цепей, заканчивающихся либо эндо-, либо экзо-двойной связью, этот многомерный подход позволил показать, что инициирующий фрагмент также может быть затронут процессом MALDI.

\section{Ионизация электрораспылением}

Метод также относится к «мягким» методам ионизации и позволяет получать ионы в газовой фазе при испарении мелкодисперсных капель в поле высокой напряжённости (рис. 2).

Особенностями данного метода является возможность работы с нелетучими соединениями и образование многозарядных ионов, которые снижают рабочий диапазон отношений массы к заряду, помогая сохранить высокое разрешение с использованием времяпролётного анализатора даже при очень больших молекулярных массах. Помимо исследований молекулярно-массовых распределений полимеров метод применяется и для изучения реакций полимеризации. Например, в работе [9] были синтезированы четыре тиокетона и была проведено ESI-MS для исследования механизма, лежащего в основе настоящего метода синтеза, который включает быстрый спиновый захват распространяющихся радикалов и последующий перекрестный обрыв с другими распространяющимися радикалами. В работе [10] блок-сополимеры поли (диметилакриламид)-b-поли(триметиленкарбонат), содержащие чувствительную к $\mathrm{pH}$ иминную связь, были успешно синтезированы путем сочетания полимеризации с раскрытием цикла (ROP) и обратимого присоединения-фрагментации. полимеризация с передачей цепи (ОПЦП). Множество блок-сополимеров с концевыми ванилиновыми группами получали путем ROP триметиленкарбоната, инициированного модифицированным ванилином, в присутствии 1,8-диазабицикло

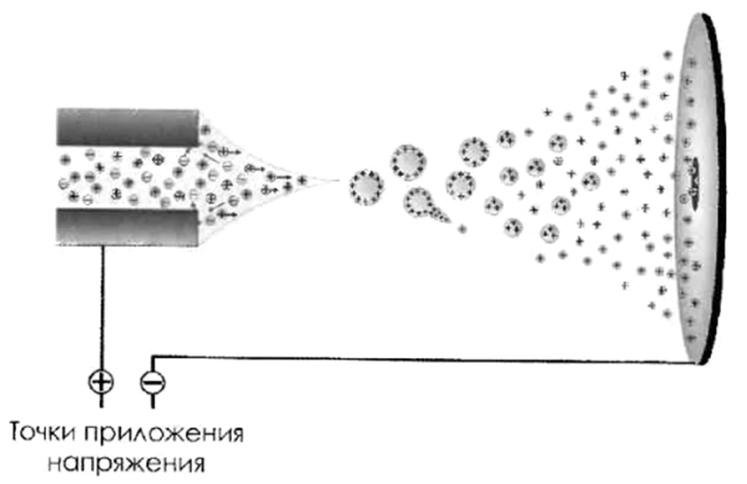

Рис. 2. Схема ионизации методом электрораспыления.

Fig. 2. The scheme of electrospray ionization. 
[5.4.0] ундец-7-ена (DBU) в качестве органо-катализатора. Анализ полученных гомополимеров с помощью масс-спектрометрии ESI-ToF (ESI-ToF-MS) показал превосходный результат по определению молярных масс. На сегодняшний день именно в таких работах применяется ионизация электрораспылением. Основным её преимуществами перед другими методами является возможность хроматографического разделения в режиме online без значительного усложнения методики. В тоже время метод требует растворения исследуемого образца, что накладывает определённые ограничения в случае исследования полимерных материалов.

\section{Другие «мягкие» методы ионизации}

Полевая десорбция - метод состоящий в нанесении образца в конденсированном состоянии или из раствора на поверхность покрытую микроиглами. На поверхность подаётся высокое напряжение, в результате чего на кончиках игл образуется поле высокой напряжённости, вызывающее ионизацию нанесённого на неё образца (рис. 3).

Например, в работе [11] представлен метод легкого удаления концевых групп полимера с использованием света, который является общим для методов контролируемой радикальной полимеризации. Процесс удаления контролировался с ис-

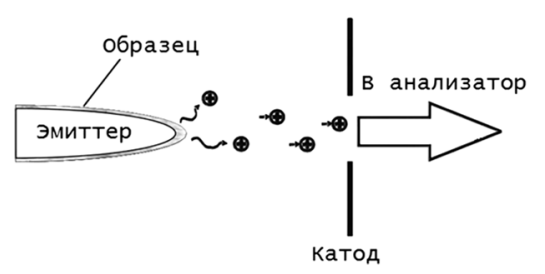

Рис. 3. Схема источника полевой десорбции.

Fig. 3. The scheme of the source of field desorption. пользованием масс-спектрометрии с полевой десорбцией. В целом десорбция полем незаменима при исследовании малополярных соединений, которые плохо ионизируются другими методами. Однако в последнее время она применяется всё реже из-за сложности пробоподготовки и плохой воспроизводимости результатов.

\section{Бомбардировка быстрыми атомами (FAB) и масс-спектромет- рия вторичных ионов (SIMS)}

Оба метода похожи по приборному исполнению: образец наносится на мишень, которая затем бомбардируется потоком частиц. Разница в том, в случае FАВ бомбардировка проводится потоком ускоренных атомов, а в случае SIMS - потоком заряженных частиц (рис. 4).

Метод FAB в настоящее время имеет довольно ограниченное применение из-за возможности анализа только полярных соединений и ограниченности массового диапазона. В тоже время метод SIMS Стал практически незаменимым для исследования полимеров. В работе [12] времяпролетная вторичная масс-спектрометрия (ToF-SIMS) широко используются для характеристики свойств поверхности и объема, в связи с тем, что физические и химические свойства на поверхности полимерных смесей могут резко отличаться от свойств в объеме из-за поверхностной сегрегации компонента с низкой поверх-

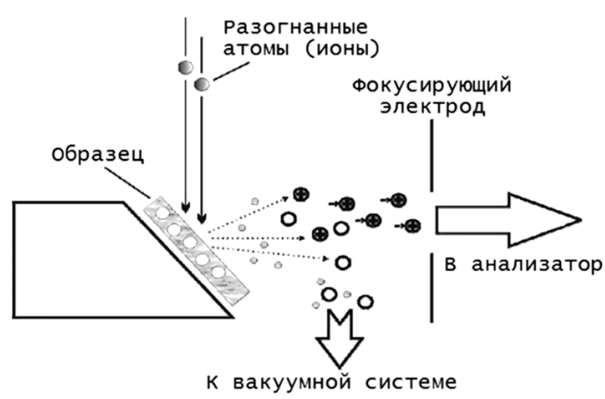

Рис. 4. Схема источника ионизации вторичными ионами быстрыми атомами. Fig. 4. The scheme of the source of ionization by secondary ions and fast atoms. 
ностной энергией. Часто SIMS применяется с современными вычислительными методам, позволяющими значительно расширить область применения. Например, в работе [13] показано, что передовые методы информатики, основанные на нейронных сетях и искусственном интеллекте могут извлекать больше информации из экспериментов по анализу поверхности, чем обычно применяемые традиционные линейные методы. Эти передовые методы позволяют надежно различать полимеры и другие материалы с очень похожими химическими структурами, чего нельзя добиться традиционными методами. Результаты работы представляют собой важный шаг в развитии новой методологии, в которой анализ данных из ToF-SIMS может проводиться полностью автоматически. Также SIMS незаменима при анализе слоёв многослойных плёнок [14] и является наиболее распространённым методом в области исследования полимеров, а именно для анализа состава, мономерного звена, концевых групп, молекулярно- массового распределения, имиджинга поверхности.

\section{Заключение}

По итогам данной статьи следует отметить, что масс-спектрометрия используется при решении большинства задач, связанных с исследованием полимеров, постепенно замещая или дополняя их. Если раньше применение масс-спектрометрии было ограничено необходимостью перевода образца в газовую фазу, то с появлением методов "мягкой” ионизации масс-спектрометрия стала применяться наравне с классическими методами. Благодаря множеству источников ионизации и способностью работать с веществами в любом фазовом состоянии, а также высокой чувствительности массспектрометрия выходит на первые позиции в анализе и физико-химических исследованиях полимеров. Наиболее востребованным вариантом для современных исследований помимо пиролитической масс-спектрометрии являются MALDI, FAB, FD и SIMS, как наиболее часто применяемый метод в области исследования поверхностей материалов и изменения их состояния от поверхности в объём.

Авторы выражают благодарность Министерству образования и науки Российской Федерации за финансовую поддержку.

\title{
Mass spectrometry as a modern method for the analysis of polymers
}

\author{
(C) 2021 Pytskii I.S., Kuznetsova E.S., Buryak A.K. \\ Frumkin Institute of Physical Chemistry and Electrochemistry, Russian Academy of Sciencesof RAS, \\ Moscow, Russian Federation
}

\begin{abstract}
The study was devoted to the investigation of the place of modern mass spectrometric methods in the polymer research. The development of methods was investigated and it was shown that practically until the end of the 20th century, the use of mass spectrometry for investigation of polymers was extremely limited due to the non-volatility of polymers. The method of pyrolytic mass spectrometry is considered as the most common method for determining the molecular weight distribution, the determination of end groups, and other general problems. More modern methods of "soft" ionization, allowing ionizing polymers from a condensed state with simultaneous desorption into the gas phase were also described. A review of the literature has shown that such methods have almost completely replaced or significantly supplemented the tools of polymeric material researchers. Matrix-assisted laser desorption/ionization (MALDI), field desorption (FD), fast atom bombardment (SIMS), and electrospray ionization (ESI) are described in the literature. Also, pyrolytic mass spectrometry with direct injection and combined with gas chromatographic separation were described as being a more classical method. It was shown that mass spectrometry is the most versatile and sensitive method for any tasks related with the analysis and investigation of polymer materials.

Keywords: mass spectrometry, polymers, pyrolysis, gas chromatography, "soft" ionization, field desorption, secondary ion mass spectrometry, surface.
\end{abstract}




\section{References}

1. Hermabessiere L., Himber C., Boricaud B., Kazour M. et al., Analytical and bioanalytical chemistry, 2018, Vol. 410, No 25, pp. 66636676.

2. Peters C. A., Hendrickson E., Minor E. C., Schreiner K. et al., Marine pollution bulletin, 2018, Vol. 137, pp. 91-95.

3. Ohtani H., Takeuchi K., Iiguni Y., Kaneko A. et al., Journal of Analytical and Applied Pyrolysis, 2017, Vol. 124, pp. 677-681.

4. La Nasa J., Biale G., Fabbri D., Modugno F., Journal of Analytical and Applied Pyrolysis, 2020, Vol. 104, pp. 38-41.

5. Crotty S., Gerişlioğlu S., Endres K.J., Wesdemiotis C. et al., Analytica Chimica Acta, 2016, Vol. 932, pp. 1-21.

6. Baumann M., Wold C., Uliyanchenko E., Weidner S. et al., International Journal of Polymer Analysis and Characterization, 2020, Vol 24, pp. 1-12.

7. Hill J.A. Surface Layer Matrix-assisted Laser Desorption Ionization Time of Flight Mass

Пыцкий Иван Сергеевич - старший научный сотрудник лаборатории физико-химических основ хроматографии и хромато-масс-спектрометрии, к.Х.н., Институт физической химии и электрохимии имени А.Н. Фрумкина РАН, Москва

Кузнецова Елена Сергеевна - старший научный сотрудник лаборатории синтеза и исследования сорбентов, к.х.н., Институт физической химии и электрохимии имени А.Н. Фрумкина РАН, Москва

Буряк Алексей Константинович - заведующий лабораторией физико-химических основ хроматографии и хромато-масс-спектрометрии, проф, д.х.н., Институт физической химии и электрохимии имени А.Н. Фрумкина РАН, Москва
Spectrometry (SL-MALDI-ToF MS) Analysis of Polymer Blend Surface Composition, 2017, Doctoral dissertation, University of Akron.

8. Chendo C., Le D., Phan T.N., Gigmes D. et al., Journal of Polymer Science Part A: Polymer Chemistry, 2016, Vol. 54, No 20, pp. 3388-3397.

9. Yu H., Shao J., Chen D., Wang L. et al., Polymer Chemistry, 2020, Vol. 11, No 18, pp. 32513259.

10. Sun J., Fransen S., Yu X., Kuckling D., Polymer Chemistry, 2018, Vol. 9, No 23, pp. 3287-3296.

11. Mattson K.M., Pester C.W., Gutekunst W.R., Hsueh A.T. et al., Macromolecules, 2016, Vol. 49, No 21, pp. 8162-8166.

12. Chan C.M., Weng L.T., Materials, 2016, Vol. 9, No 8, pp. 655-657.

13. Madiona R.M., Winkler D.A., Muir B.W., Pigram P.J., Applied Surface Science, 2019, Vol. 478, pp. 465-477.

14. Zhou C., Sun D., Garcia R., Stevie F.A., Analytical Methods, 2018, Vol. 10, No 21, pp. 2444-2449.

Pytskii Ivan S. - PhD (chemistry), laboratory of physical-chemical basics of chromatography and chromato-mass-spectrometry, Institute of Physical chemistry and electrochemistry, Moscow. E-mail: ivanpic4586@gmail.com

Kuznetsova Elena S. - PhD (chemistry), laboratory of synthesis and investigation of sorbents, Institute of Physical chemistry and electrochemistry, Moscow

Buryak Alexey K. - prof., grand $\mathrm{PhD}$ (chemistry), laboratory of physical-chemical basics of chromatography and chromato-mass-spectrometry Institute of Physical chemistry and electrochemistry, Moscow. 